\section{Modafinil for fatigue in multiple sclerosis}

\section{E Willoughby}

\author{
Single daily dose of modafinil is of benefit
}

F atigue is a perplexing and at times incapacitating symptom in multiple sclerosis. It has both physical and cognitive components and its severity is not closely related to the extent of neurological disability, nor to clinical or laboratory measures of inflammatory activity, nor to depression. ${ }^{12}$ Treatment is unsatisfactory, with few controlled studies and with interpretation of results hindered by somewhat blunt clinical scales to measure effects. Evidence is accumulating that fatigue in multiple sclerosis is due to dysfunction of the $\mathrm{CNS}^{3{ }^{3}}$ but lack of understanding of the underlying pathophysiology makes a rational approach to treatment difficult. The paper by Rammohan $e a^{5}$ (this issue, pp 179-183) is a useful addition to the limited literature on the subject. It demonstrates improvement in fatigue in patients with multiple sclerosis taking $200 \mathrm{mg}$ modafinil a day.

Modafanil stimulates wakefulness and, compared with amphetamines, has a more selective action in the brain and fewer side effects. It is an $\alpha$ - 1 adrenergic agonist with complex actions also on other neurotransmitter systems. A relatively long half life makes single daily dosing practicable. Interest in its effect on fatigue was stimulated by a study showing effectiveness in reducing daytime sleepiness in narcolepsy-at the same time measures of fatigue were improved. There is clearly overlap between fatigue and sleepiness, but in many patients the symptoms can be distinguished reasonably well.

Limitations of the present report in patients with multiple sclerosis are the single blind design, a standard dose titration sequence for modafinil and placebo and, especially, the short time on active treatment. Although $200 \mathrm{mg}$ modafinil a day was of benefit, a higher dose of 400 mg was not. Increased side effects on the higher dose is a more likely explanation than loss of responsiveness to the treatment after 2 weeks. It was noted that some patients did better on the higher dose, and it would be expected that there may be considerable variation among patients in the doses producing both side effects and improvement in symptoms.

Further studies on modafinil for fatigue in multiple sclerosis are warranted, with the need for a double blind study assessing treatment for longer periods, with titration of the dose for individual patients. Potential for habituation with modafinil seems relatively low but needs careful assessment.

J Neurol Neurosurg Psychiatry 2002;72:150

\section{Author's affiliation}

E Willoughby, Department of Neurology, Auckland Hospital, Park Road, Private Bag 92024, Auckland 1, New Zealand; ErnieW@adhb.govt.nz

\section{REFERENCES \\ 1 Giovannoni G, Thompson A J, Miller D H, et al. Fatigue is not associated with raised inflammatory markers in multiple sclerosis. Neurology 2001;57:676-81. \\ 2 Bakshir, Shaikh Z A, Miletich R S, et al. Fatigue in multiple sclerosis and it's relationship to depression and neurologic disability. Mult Scler 2000;6:181-5. \\ 3 Leocani L, Colombo B, Magnani G, et al. Fatigue in multiple sclerosis is associated with abnormal cortical activation to voluntary movement: EEG evidence. Neuroimage 2001;13:1186-92. \\ 4 Colombo B, Boneschi F M, Rossi P, et al. MRI and motor evoked potential findings in nondisabled multiple sclerosis patients with and without symptoms of fatigue. J Neurol 2000;247:506-9. \\ 5 Rammohan KW, Rosenberg JH, Lynn DJ, et al. Efficacy and safety of modafinil (Provigil ${ }^{\circledR}$ ) for the treatment of fatigue in multiple sclerosis: a two centre phase 2 study. J Neurol Neurosurg Psychiatry 2002;72:179-183.}

\title{
Community based rehabilitation
}

\section{R J Hardie}

\section{Community based rehabilitation after severe traumatic brain injury}

$\mathrm{P}$ ractitioners of rehabilitation are often criticised for failing to evaluate their clinical work critically and assembling sound research evidence upon which to base it. The paper by Powell et al (this issue, pp 193-202) describes the results of an extremely ambitious and almost unique interventional study of community based rehabilitation in around 100 patients after moderate or severe traumatic brain injury, following the gold standard methodology of a randomised controlled trial.

At entry to the study, patients were allocated to either active multidisciplinary community rehabilitation, or a single therapist visit with the provision of written information about available resources, effectively a control group. Overall, despite the marked heterogeneity of the participants, the two comparison groups were well matched. The results showed significant improvements after active intervention for several hours a week for an average of 6 months, when followed up by a blinded assessor some 2 years later.

Considering the amount of work involved, both in providing the therapeutic input and gathering the outcome data, the therapeutic effect size may at first sight seem nevertheless disappointing. Randomised controlled trials are all very well in certain settings, but may not always be necessary or appropriate in evaluating rehabilitation, where benefits may be modest and multifactorial. ${ }^{2}$ One of the two primary outcome measures in the study was the original Barthel index. Although widely used in clinical practice as a rapid and easy measure of activities of daily living, it is notorious for its ceiling effect and has since been modified in an attempt to improve it. Both groups had a median score of $100 \%$, with negative as well as positive changes at follow up. The improvement of one or two points at the upper limit of the index is of rather doubtful functional, as opposed to statistical, significance.

Fortunately, there was also significant improvement measured by the second primary outcome measure used by the authors, the brain injury community rehabilitation outcome (BICRO)-39 scale. This showed much more meaningful and impressive benefits of active intervention, with greater relevance, measuring what subjects really do, rather than can do, in their homes including such areas as social participation, productive employment and psychological wellbeing. 
Powell et al ${ }^{1}$ acknowledge that they could only evaluate the overall framework for delivering rehabilitation rather than its specific components. They have offered important new evidence to support the wider development of community based multidisciplinary rehabilitation teams. Wilson ${ }^{3}$ has reminded us that it is wasteful spending hours delivering unevaluated therapy that may be of little or no benefit to a patient. The challenge now is to find evidence based ways of determining more precisely what these teams should actually do, when, and for how long.

Competing interestes: none declared

J Neurol Neurosurg Psychiatry 2002;72:150-151

Author's affiliation

Author's affiliation
R Hardie, Department of Neurology,

St George's and Atkinson Morley's Hospitals, London, UK and Wolfson Neurorehabilitation Centre, London, UK;

Richard.Hardie@ccmail.stgh-tr.sthames.nhs.uk

\section{Which CT features help predict outcome after head injury?}

\section{J Wyper}

\section{Presence of subarachnoid blood and overall appearance are helpful}

is $\mathrm{n}$ the paper by Wardlaw et al (this issue, pp 188-192) ${ }^{1}$ it is shown that the presence of subarachnoid blood and "overall appearance" of a CT scan could be added to the already established variables of age, Glasgow coma score, and pupil reaction to improve prediction of 1 year survival after head injury. This has been a thorough investigation and should be of practical benefit in improving the predictive model. A deficiency, which is recognised by the authors, is that the interrater variability in classifying the overall appearance of scans has still to be established. It remains to be seen whether or not this classification proves to be sufficiently robust for day to day application within increasingly busy neuroradiology departments.

Although this paper has focused on CT, MRI may be a better imaging modality for prognostic estimation, especially on account of its ability to detect brain stem lesions. ${ }^{2}$ If the current constraints around access for patients with acute head injury at the time of admission can be tackled then there could be a gradual shift towards MRI.

\section{REFERENCES}

1 Powell J, Heslin J, Greenwood R. Community-based rehabilitation after severe traumatic brain injury: a randomised controlled trial. J Neurol Neurosurg Psychiatry 2001;72;193-202.

2 Andrews K. The limitations of randomised controlled trials in rehabilitation research. Clin Rehabil 1992;5:5-8.

3 Wilson B. Research and evaluation in rehabilitation. In: Wilson B, McLellan DL. Rehabilitation studies handbook. Cambridge: University Press 1997:161-87.
It is also clear that more subtle outcome measures are difficult to predict. Neurophychiatric status after mild head injury has been investigated ${ }^{3}$ and variables including $\mathrm{CT}$ failed to predict outcome.

J Neurol Neurosurg Psychiatry 2002;72:151

\section{Author's affiliation}

D J Wyper, Department of Clinical Physics, South Glasgow University Hospitals NHS Trust, Glasgow, UK

\section{REFERENCES}

1 Wardlaw JM, Easton VJ, Statham P. Which $C T$ features help predict outcome after head injury? J Neurol Neurosurg Psychiatry 2001;72:188-92.

2 Firsching $\mathbf{R}$, Woischneck D, Klein S, et al. Classification of severe head injury based on magnetic resonance imaging. Acta Neurochir (Wien) 2001;143:263-71.

3 McCullagh S, Oucherlony D, Protzner A, et al. Prediction of neuropsychiatric outcome following mild trauma brain injury: an examination of the Glasgow Coma Scale. Brain Inj 2001;15: 489-97. 